IX.

\title{
Ludwig Traube und die Geschichte der experimentellen Pathologie in Deutschland ${ }^{1}$ ).
}

\author{
(Kritische Bemerkungen.) \\ Von \\ H. Boputtau (Berlin).
}

Im Januar 1846 liess Ludwig Traube das erste Heft seiner „Beiträge zur experimentellen Pathologie und Physiologie " erscheinen, in dessen Einleitung, die in Vorwort seiner 1871 gesammelten Abhandlungen wieder abgedruckt ist, er die Lücke beklagt, die damals zwischen den gründlichen pathologisch-anatomischen und klinischen Beobachtungen der verdientesten Forscher klaffte und durch Deduktion und Hypothese ausgefüllt werde, statt systematisch und zielbewusst den Weg des Experimentes zu beschreiten, den in Frankreich schon vor längerer Zeit Magendie gewiesen hatte, dessen Methode aber nicht mehr dem damaligen Standpunkte genüge. Von jenen Beiträgen sind nur zwei Hefte erschienen. Schon im nächsten Jahre begründeten die Mitherausgeber derselben Rudolf Virchow und Benno Reinhardt das "Archiv für pathologische Anatomie und Physiologie und für klinische Medizin", dessen noch heate jährlich zunehmende Bändezahl mit reichem Inhalt von dem $\Lambda$ ufschwunge Zeugnis gibt, den seit jenen Tagen die deutsche Pathologie - allgemein und zusammenfassend ausgedrückt - gcnommen hat. In dem Prospekte zu diesem Archiv hiess es: „Die praktische Medizin als die angewendete theoretische, die theoretische als pathologische Physiologie ist das Ideal, dem wir, soweit es unsere Kräfte gestatten, zustreben werden. Die pathologische Anatomie und die Klinik, obwohl wir ihre Berechtigung und Selbständigkeit vollkommen anerkennen, gelten uns doch vorzugsweise als die Quellen für neue Fragen, deren Beantwortung der pathologischen Physiologie zufällt. Da aber diese Fragen zum grossen Teile erst durch ein mühsames und umfassendes Detailstudium der Erscheinungen an Lebenben und der Zustände an der Leiche formuliert werden müssen, so setzen wir eine genaue und bewusste Entwicklung der anatomischen und klinischen Erfahrungen als die erste und wesentlichste Forderung der Zeit. Aus einer solchen Empirie resultiere dann allmählich die wahre Theorie der Medizin, die pathologische Physiologie". Diese Worte,

1) Vortrag, gehalten in der Berliner Gesellschaft für Geschiohte der Naturwissenschaften und Medizin am 15. Februar 1918. 
in denen das pathologische Experiment als solches überhaupt nicht einmal genannt wird, sind vor heute über 70 Jahren niedergeschrieben, ein Jahr vor der ersten Einführung der graphischen Methode in die normale Physiologie durch Karl Ludwig, vor der ersten Verzeichnung des Ablaufs der Muskclzusammenzichung und vor der ersten Messung der Nervenleitungsgeschwindigkeit durch Holmholtz, vor der Mehrzahl der physikalisch- und chemisch-physiologischen und durch das Tierexperiment gewonnenen Errungenschaften, welche aus der normalen Physiologie, wie sie uns in Johannes Müller's sicher unsterblichem Handbuche entgegentritt, das gernacht haben, was sie heute ist und wodurch sie alle Zweige der theoretischen und praktischen Hedizin so hat befruchten können, wie es in allen Kulturländern der Fall ist, und zwar bei uns in Deutschland, ohne dass bis auf den heutigen Tag auch nur ein einziger ordentlicher Lehrstuhl und ein einziges selbständiges Universitätsinstitut für experimentelle Pathologie bzw. pathologische Physiologie existiert! Gewiss hat Virchow, der den Begriff der pathologischen Physiologie so hoch gestellt und so weit gefasst hat, selbst experimentiert und dem pathologischen Experiment die wünschenswerte Achtung gewährt, er hat der chemischen Pathologie eine der ersten Pflanzstätten geschaffen; aber zwei Umstände, die sich an seine Person knüpfen, haben der Selbständigmachung der experimentellen Pathologie hindernd im Wege gestanden: dem Schöpfer der Zellularpathologie stand die Erkenntnis des Vorgangs in der krankhaft veränderten Zelle als zu erreichendes Ideal vor Augen, dem vor allem mit dem Rüstzeug des Mikroskops, der histologischen Technik und Mikrochemie beizukommen ist, und für den Meister der pathologischen Anatomic und vielseitigen Forscher und Arbeiter, den hygienische und sozial- wie allgemeinpolitische, anthropologische und prähistorische Fragen beschäftigten, lag die Personalunion der pathologischen Anatomie und Physiologie so nahe und musste ihm so selbstverständlich erscheinen, wie es zwischen der normalen Anatomie und Physiologie als Forschungs- und Lehrgebiete der Hochschulen Jahrhunderto möglich gewesen war und lange ins neunzehnte Jahrhundert hinein noch blieb, wie sie auch zwischen normaler und pathologischer Anatomie bis damals vielfach bestanden hatte, wo Johannes Müller die Abtrennung der letzteren vom eigenen Lehrstuhle und Errichtung des neuen gerade für seinen ausgezeichneten Schüler Virchow befürwortete!

So ist es gekommen, dass wir als den Begründer der experimentellen Pathologie in Deutschland Ludwig Traube ohne Einschränkung zu bezeichnen haben, dessen 100. Geburtstag kürzlich mit vielfachen Gedenkaufsätzen in Erinnerung gebracht wurde, in denen naturgemäss noben dem experimentellpathologischen das Verdienst Traube's als des unvergleichlichen Klinikers besonders betont werden musste: Ueber dem, was or als Arzt und klinischer Lehrer geschaffen hat, soll aber eben nicht vergessen werden, dass diese Leistungen grossenteils auf seinen Experimentalergebnissen beruhten. Nichts kann diesen Zusammenhang besser erkennen lassen, als das Studium seiner gesammelten Abhandlungen, in deren erstem Band sich nur Experimentaluntersuchungen finden, nämlich: die klassische Untersuchung, durch welche das Wesen der nach 
beiderseitiger Vagusdurchschncidung am Halse bei Säugetieren auftretenden tödlichen Lungenveränderungen als Schluckpneumonie nachgewiesen wurde; die Untersuchungen über dyspnoische Atmung, insbesondere dyspnoische Vagusreizung; die hämodynamische Lntersuchung (vermittels des nicht lange zuvor durch K. Ludwig erfundenen Kymographions) der Wirkungsweise der Digitalis beim Warmblüter; Versuche über die Wirkung von Kurare und Nikotin, der gallensauren Salze und des Kaliumnitrats auf Herz und Kreislauf; Versuche über die Wirkungsweise des Kohlenoxyds, sowie der Rolle von Kohlensäureüberschuss und Sauerstoffmangel im Blute beim Zustandekommen der Dyspnoe; und manches andere. Und wenn man die klinischen Bände der Abhandlungen durchforscht, so bogegnet einem allerorten, ob nun in der meisterhaft behandelten Kasuistik oder in den theoretischen Aufsätzen - Theoric des Fiebers, der Hämaturie, der Digitaliswirkung usw. - immer wieder die Bezugnahme auf die Physiologie und das pathologische Experiment; diese befruchten seine zahlreichen Beiträge zum Fortschrift der Diagnostik, insbesondere der physikalischen durch Deutung der Herz- und Gefässgeräusche, Verbesserung der damals noch jungen Sphygmo- und Kardiographie usw. Das rein Chemische lag ihm wohl ferner, obschon er durch seine schon erwähnten grundlegenden Arbeiten über Digitaliswirkung u. a. den Grundstein zur experimentellen Arzneimittellehre gelegt hat; wie hier chemisches Arbeiten und Tierversuch Hand in Hand zu greifen haben, hatte, wie Claude Bernard in Frankreich, inzwischen für das deutsche Sprachgebiet Rudolf Buchheim in Dorpat gezeigt, der aus Privatmitteln dort das erste pharmakologische Institut eingerichtet hatte und Anfang der 70er Jahre mit Karl Ludwig's Schüler Schmiedeberg das Archiv für experimentelle Pathologie und Pharmakologie gründete. Den therapeutischen Erfolgen, welche das Handinhandarbeiten der deutschen chemischen Forschung mit dem Tierversuch zeitigte, war es zu danken, dass allmählich an allen deutschen Universitäten pharmakologische Institute und Lehrstühle cingerichtet wurden. Diese haben nun sicher zum grossen Teil in weiterem Sinne experimentelle Pathologie getrieben und die pathologische Physiologie im Sinne Virchow's ausbauen helfen - ebenso wie auf der anderen Seite hervorragende Kliniker - ich nenne nur Naunyn, der nach Buchheim's Tode die Mitredaktion des Archivs für experimentelle Pathologie übernahm, v. Krehl: zahlreiche nicht minder grosse Meister der Klinik in und ausserhalb des Reichs, ja selbst praktische Aerzte experimentierten und experimentieren rastlos: leider haben aber weitere Umstände dazu beigetragen, dass bei uns der experimentellen Pathologie die offiziellen Lehr- und Pflegestätten immer noch fehlen: als einfacher Kroisarzt hat seinerzeit Robert Koch, chemisch-experimentell ein Schüler des Physiologen Georg Meissner, die grundlegende Methodik zur Erforschung der krankheitserregenden Spaltpilze geschaffen. Ihre Verbesserung und praktische insbesondere prophylaktische Anwendung führte zur Schaffung der hygienischen Institute, in denen der Natur der Sache nach auch anderweitig - auch pathologisch - experimentiort werden muss. Die aus der Bakteriologic sich entwickelnde Immunitätslehre und Serologie mit ihrer an sich rein 
experimentell-pathologischen Mcthodik fand bei uns im Reichihre Pflegestätten teils eben in den hygicnischen Instituten, in neuester Zeit noch in den zwei Instituten für experimentelle Therapie, wie dieser Zweig der Dynamik noch nicht isoliert daryestellter Pharmaka und Toxika genannt wird, dessen Abgrenzung oder gegenseitiges Verhältnis zur alten Pharmakologie sogar schon polemisch bekandelt worden ist! So düren wir fast sagen, dass es bei uns zu einer Zersplitterung der experimentellpathologischen Forschung und Vernachlässigung ihres Unterrichts gekommen ist, der um so mehr bedauert werden muss, als es in den anderen Kulturländern meistens besser darum bestellt ist: In Oesterreich haben Männer wie Knoll und Stricker, die beide nicht mehr unter den Lebenden weilen, schon vor Jahren Lehrstühle und Institute unseres Wissenszweiges erhalten, die unter ihren Nachfolgern sich vervielfacht haben, und auch in Itatien, England, Amerika und den skandinavischen Ländern bestehen an vielen Universitäten solche Lehrstühle und Institute neben denjenigen für normale Physiologie, für pathologische Anatomie, für Pharmakologie, für Hygiene, ja selbst für experimentelle Therapie (letztere bisweilen für den Begriff der Pharmakologie gesetzt!). Ihre Forschungsaufgabe ist klar, ihre Lehraufgabe ist die systematische Unterweisung der Heilkunde Studierenden in der pathologischen Physiologie, ein Unterricht, der die fehlende Brücke zu schlagen hat zwischen dem Unterricht in der Physiologie und der Klinik und praktischen Schulung am Krankenbette, der das Erkennen der pathologisch gestörten, veränderten Funktion zu erleichtern hat durch Vergleichung mit der normalen und Erläuterung des Mechanismus dieser Veränderungen. Dieser Aufgabe kann weder der diagnostische Kurs noch auch die sogenannte propädeutische Klinik genügen, deren Vertreter allerdings, wie es ja auch Traube war und getan hat, auf die Ergebnisse des Experimentes, auf die normale und pathologische Physiologie zurückzugreifen hat, im wesentlichen aber doch die Beobachtung und Behandlung des kranken Menschen zu lehren hat. Ein Institut für experimentelle Pathologie wird darum nicht ohne Beziehung zu diesem letzteren bleiben dürfen, ganz im Gegenteil: es soll ihm eine Einrichtung angegliedert sein, die man als Versuchsklinik, als Anstalt zur Beobachtung und Behandlung solcher Kranken wird bezeichnen dürfen, an denen eine Bereicherung pathologischer Erkenntnis zu erwarten ist, und die ihr aus dem Krankenbestande anderer Kliniken und der Privatpraxis zu diesem Zwecke zuzuweisen sind. An diesen Kranken werden die zum Fortschritt der Theorie und Praxis notwendigen "Versuche" neuartiger Behandlung vorzunehmen sein, über die vielfach gestritten und die vielfach verurteilt worden sind: selbstverständlich mit besonderer Zustimmung der Kranken, die Gefühl für die wissenschaftliche Bedeutung ihres Falles und die. Möglichkeit, dass für sie selbst und andere Nutzbringendes gefunden werde, besitzen, und deren Pflege und Beschwerdeverminderung alle nur irgend mögliche Aufmerksamkeit zuzuwenden ist. Solche Einrichtungen sind übrigens keineswegs neu, sondern in manchen Ländern schon vorhanden und als zweckmässig erkannt: Bei uns ist das Institut für Infektionskrankheiten "Robert $\mathrm{Koch}^{"}$ von Anfang an im Sinne solcher 
148 H. Boruttau, Ludwig Traube u. die Geschichte der experimentellen Pathologio.

Vereinigung experimenteller Pathologie und Versuchs- und Musterklinik angelegt worden.

Dafür, dass endlich die experimentelle Pathologie auch bei uns aufhöre, Stiefkind des Hochschulunterrichts zu sein, ist vor und während des Krieges mebrfach eingetreten worden, insbesondere von Virchow's bisherigem Nachfolger, Orth. Es ist dringend zu wüschen, dass das allgemeine Versprechen, dass die nach diesem Kriege nötige Sparsamkeit nicht notwendige Kulturaufgaben schädigen soll, insofern erfüllt werde, als die Errichtung der Universitätsinstitute für experimentelle Pathologie nun endlich, wenigstens allmählich in Angriff genommen wird. 